Proceedings

\title{
Analysis of Synthesis Mechanism of Gold Nanoparticles Using Glass Microfluidics ${ }^{\dagger}$
}

\author{
Yuanwei Wang ${ }^{1}$, Yu Tanabe ${ }^{2}$ and Hiromasa Yagyu ${ }^{1, *}$ \\ 1 Department of Mechanical Engineering, Kanto Gakuin University, Yokohama 236-8501, Japan; \\ m18J1002@kanto-gakuin.ac.jp \\ 2 Fukoku Co., Ltd., Ageo 362-8561, Japan; m16J1004@kgu.ac.jp \\ * Correspondence: yagyu@kanto-gakuin.ac.jp; Tel.: +81-45-786-7118 \\ † Presented at the Eurosensors 2018 Conference, Graz, Austria, 9-12 September 2018.
}

Published: 3 December 2018

\begin{abstract}
According to LaMer diagram, a clearly separate of nucleation and grows step is required to synthesis of monodisperse nanoparticles. However, a critical mixing time $T_{c}$ until the growth process is started, is not clear experimentally. In this paper, we prepared gold nanoparticles (GNPs) by liquid-phase reduction using citric acid on microfluidics with different flow rates. From relationship of the diameter of the prepared nanoparticle and the mixing time, $T_{c}$ for the preparation of monodisperse GNPs was found for the first time.
\end{abstract}

Keywords: nanoparticles; FWHM; absorption

\section{Introduction}

In an application of metallic nanoparticle to bio/chemical sensor with localized surface plasmon resonance [1], there are strong demands for monodisperse nanoparticles with coefficient of variation (C.V.) defined as the ratio of the standard deviation to the mean diameter smaller than 0.1. The synthesis mechanism of nanoparticles by liquid-phase reduction was divided into three stages by LaMer's model [2]: (I) the number of particles (atoms) increases continuously. (II) When the number of atoms exceeds the critical supersaturation $\left(C_{m i n}\right)$, the atoms aggregate to form crystal nuclei, and the number of particles begins to decrease. (III) After a large number of atoms are consumed, the number of particles would gradually decline to below the critical supersaturation level $\left(C_{m}\right)$. The number of particles no longer increases, and the growth of nuclei dominates the process (Figure.1). To synthesize monodisperse nanoparticles, the reagents must be uniformly mixed at the molecular level before stage III is started, and stage II and III have to be clearly divided. Therefore, we expect that a critical mixing time $T_{c}$ exists in the synthesis process. In this paper, we fabricate glass microfluidic device with simple $\mathrm{Y}$ shaped microchannel and analyzed $T_{c}$ for synthesis of monodisperse GNPs by evaluating particle diameter.

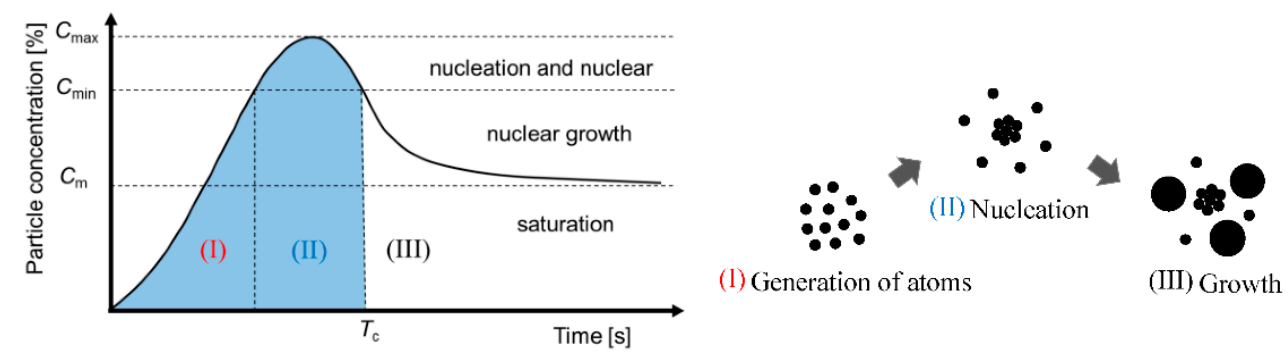

Figure 1. LaMer diagram. $T_{c}$ is a critical mixing time for preparation of monodisperse nanoparticles. 


\section{Materials and Methods}

\subsection{Glass Device}

Soda-lime glass substrates $(25 \mathrm{~mm} \times 76 \mathrm{~mm} \times 1.2 \mathrm{~mm})$ were processed by micropowder blasting [3]. A Y-shaped microchannel was processed on the bottom glass substrate. Three holes were processed on the upper glass substrate. The fabrication process can be outlined as follows. A dry film resist (MS7050, Mitsubishi Paper Mills Ltd., Tokyo, Japan) was coated on the bottom substrate, and a mask pattern with a Y-shaped channel was then fabricated via photolithography. Subsequently, $\mathrm{Al}_{2} \mathrm{O}_{3}$ microparticles with a mean diameter of $25 \mu \mathrm{m}$ were accelerated from the nozzle with a diameter of $0.8 \mathrm{~mm}$ toward the sample with high-pressure $(0.3 \mathrm{MPa})$ airflow and an incident angle of $90^{\circ}$. In order to process the entire surface of the glass substrate, the substrate was repeatedly scanned by the $X-Y$ stages at a constant velocity of $1000 \mathrm{~mm} / \mathrm{min}$ and a moving step of $5 \mathrm{~mm}$. On the other hand, two inlet holes and one outlet hole on the upper substrate were processed by a router with a diameter of $3 \mathrm{~mm}$. The two fabricated glass substrates were immersed in dilute $\mathrm{NH}_{4} \mathrm{OH} / \mathrm{H}_{2} \mathrm{O}_{2}$ solution $\left(\mathrm{NH}_{3} \mathrm{OH}: \mathrm{H}_{2} \mathrm{O}_{2}: \mathrm{H}_{2} \mathrm{O}=1: 1: 5\right)$ for $24 \mathrm{~h}$ to form a sealed channel. Finally, they were thermally bonded in the oven at $610^{\circ} \mathrm{C}$ for $8 \mathrm{~h}$ and cooled at the rate of $30{ }^{\circ} \mathrm{C} / \mathrm{min}$ under a pressure of $3.7 \mathrm{kPa}$. The two stacked glass substrates were sandwiched by two polished alumina plates to keep the glass surface smooth. In this study, the devices with channel widths of $260 \mu \mathrm{m}$ (70 $\mu \mathrm{m}$ depth) was fabricated (Figure2a).

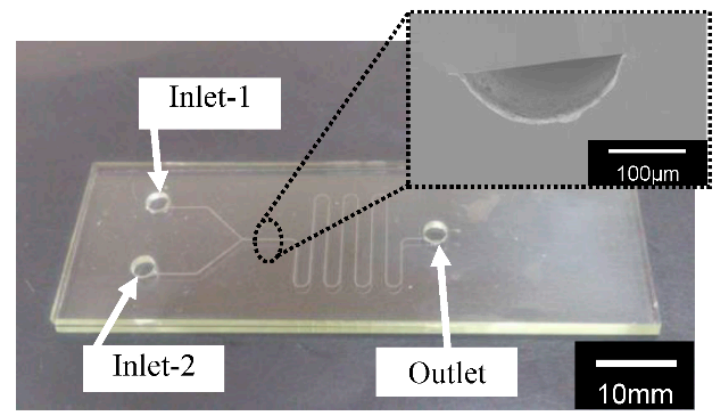

(a)

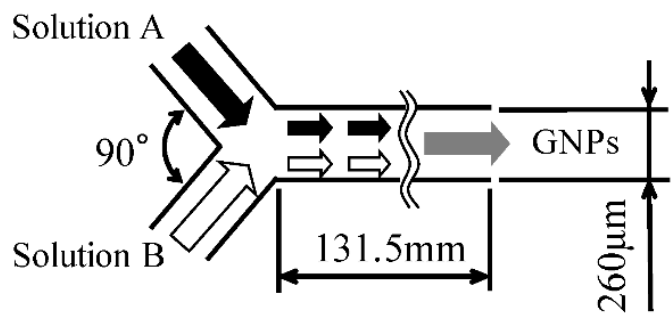

(b)

Figure 2. (a) Photograph of the fabricated glass microfluidic device. (b) Schematic image of a joint section in the microchannel.

\subsection{Synthesis}

Table 1 shows the reagents for the synthesis of GNPs. An aqueous solution of tetrachloroaurate (III) tetrahydrate (solution-A, $0.368 \mathrm{mM}$ ) was used as a source of $\mathrm{Au}$ ions. Aqueous solutions of sodium citrate acid $(33.7 \mathrm{mM})$ and tannic acid $(5.82 \mathrm{mM})$ were used as the reducing agent and protective agent, respectively. Solution-A $(5 \mathrm{~mL})$ was injected into Inlet-1, and a mixture of $4.0 \mathrm{~mL}$ reducing agent solution, $0.1 \mathrm{~mL}$ protective agent solution, and $75.9 \mathrm{~mL}$ distilled water (solution-B, 5 $\mathrm{mL}$ ) was injected into Inlet- 2 with a syringe pump at the flow rate of 0.01 to $0.1 \mathrm{~mL} / \mathrm{min}$ (Figure $2 \mathrm{~b}$ ). In this study, the mixed solution in the device was collected in a bottle through the silicone tube. The absorption spectrum of the GNPs synthesized by the batch process remained unchanged after 1 week. After 1 week at the room temperature from the synthesis on the device, the visible-light absorption spectrum of the solution was measured for a qualitative evaluation of the size distribution of the nanoparticles. This study focuses on mixing before the growth process. The total mixing time, including the mixing time in the device and out of device need for synthesis of GNPs is constant for all flow rates and channel width. Therefore, by evaluating the time variation of the absorption spectrum of the solution in the batch process, we can confirm that the synthesis of GNPs was completed. Table 1 shows the reagents for the synthesis. 
Table 1. The reagents for the synthesis.

\begin{tabular}{ccc}
\hline Reagent & Solution-A & Solution-B \\
\hline $\mathrm{HAuCl}_{4} \cdot 4 \mathrm{H}_{2} \mathrm{O}$ & $1 \mathrm{~g}$ & - \\
Sodium citrate acid $(33.7 \mathrm{mM})$ & - & $4.0 \mathrm{~mL}$ \\
Tannic acid $(5.82 \mathrm{mM})$ & - & $0.1 \mathrm{~mL}$ \\
$\mathrm{H}_{2} \mathrm{O}$ & $79 \mathrm{~mL}$ & $75.9 \mathrm{~mL}$ \\
\hline
\end{tabular}

\subsection{Characterization}

The spectra of GNPs after 1 week from the synthesis were measured using a UV-vis spectrometer (UV-1240, Shimadzu Corporation, Kyoto, Japan), and the full width at half maximum (FWHM) and absorption wavelength at the peak were calculated. A small FWHM of the absorption peak resulted in a small size distribution of nanoparticles, and a small peak wavelength was induced by a small mean diameter. The synthesized GNPs were observed using transmission electron microscopy (TEM) (JEM-2010, JEOL Ltd., Tokyo, Japan) to evaluate dispersibility and nanoparticle diameter.

\section{Results and Discussion}

\subsection{Absorption Spectrum and Mixing Time}

Figure 3a shows FWHM and absorption wavelength at the peak as a function of the flow rate. In the absorption spectrum of the synthesized GNPs, FWHM at the peak showed minimum value at the flow rate of $0.03 \mathrm{~mL} / \mathrm{min}$. The peak wavelength decreased at $0.05 \mathrm{~mL} / \mathrm{min}$. FWHM and absorption wavelength of the synthesized GNPs by the $100 \mathrm{~mL}$ beaker are $94.9 \mathrm{~nm}$ and $538 \mathrm{~nm}$.

Figure $3 \mathrm{~b}$ shows FWHM and absorption wavelength at the peak as a function of mixing time. In this study, the mixing time $T$ was calculated from the flow velocity $v$ and the channel length $L$ using $T=L / v=L S / Q$. The flow velocity $v$ was calculated from the flow rate $Q$ of syringe pump and crosssectinl area of the channel $S$, and the area was calculated as $1.3 \times 10^{-8} \mathrm{~m}^{2}$ in the case of a semi-circular channel section, as shown in Figure 2a. At the flow rate of $0.03 \mathrm{~mL} / \mathrm{min}$, which showed minimum FWHM, the mixing time was calculated as $1.7 \mathrm{~s}$ from the semi-circular cross-sectional area of the microchannel and channel length $(131.5 \mathrm{~mm})$ after Y-shapejunction.

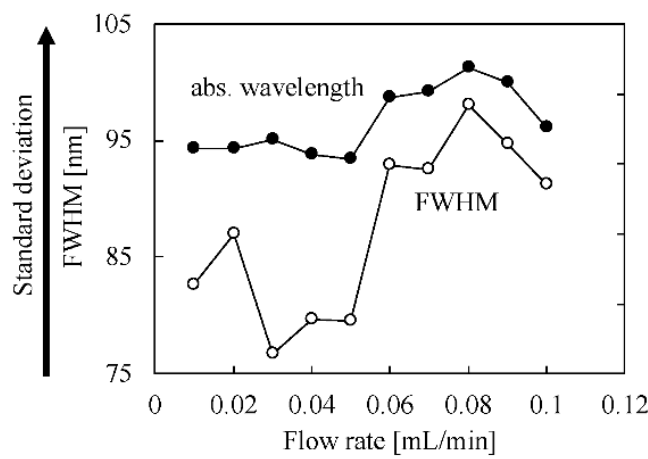

(a)

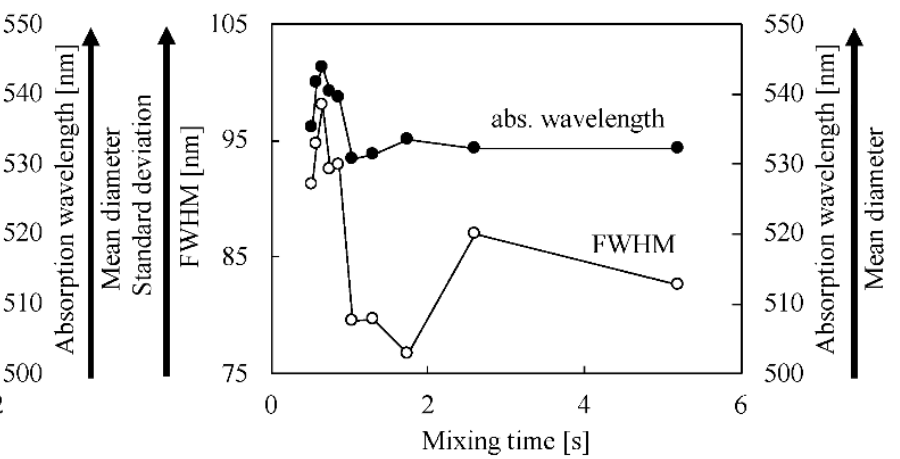

(b)

Figure 3. (a) The full width at half maximum (FWHM) and absorption wavelength at the peak as a function of the flow rate. (b) FWHM and absorption wavelength at the peak as a function of mixing time.

\subsection{Particle Size Distribution}

Figure 4 shows TEM images of the synthesized gold nanoparticles. From TEM images, it was found that monodisperse GNPs with mean diameter of $11.5 \mathrm{~nm}$ and C.V. of 0.09 was synthesized at the flow rate of $0.05 \mathrm{~mL} / \mathrm{min}$ as shown in Figure 5. Mean diameter and standard deviation of the 
synthesized GNPs by the $100 \mathrm{~mL}$ beaker are $17.6 \mathrm{~nm}$ and $3.85 \mathrm{~nm}$. These results indicated that $T_{c}$ is defined as $1.7 \mathrm{~s}$, and the synthesis mechanism is subjected to LaMer diagram.



Figure 4. TEM images of the synthesized GNPs by the device at the flow rate of $0.01,0.05,0.1 \mathrm{~mL} / \mathrm{min}$, and by $100 \mathrm{~mL}$ beaker.

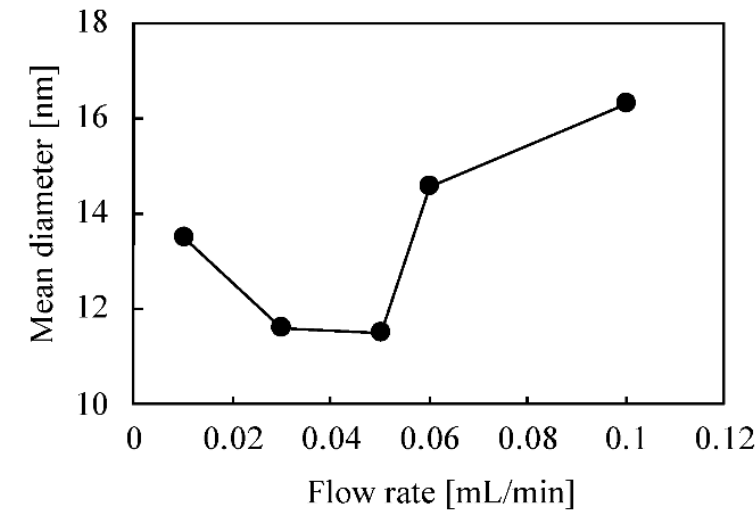

(a)

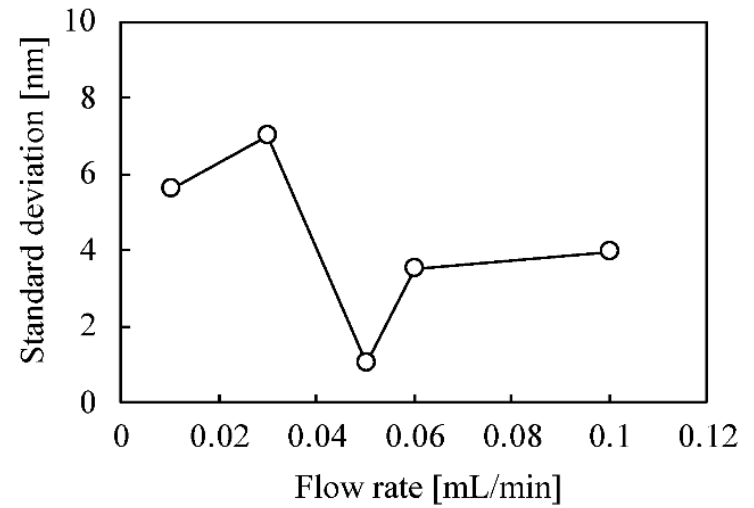

(b)

Figure 5. (a) Mean diameter and (b) standard deviation as a function of the flow rate.

\section{Conclusions}

We have demonstrated the synthesis of GNPs in the simple Y shaped microchannel device, and the characterization of synthesized GNPs. The relationship of the diameter of the prepared nanoparticle and the mixing time confirmed that the critical mixing time $T_{c}$ for monodisperse GNPs was existed in the synthesis process.

Acknowledgments: The present study was supported in part by the Tanaka Kikinzoku Memorial Foundation.

Conflicts of Interest: The authors declare no conflict of interest. The founding sponsors had no role in the design of the study; in the collection, analyses, or interpretation of data; in the writing of the manuscript, and in the decision to publish the results.

\section{References}

1. Bolduc, O.R.; Masson, J.F. Advances in Surface Plasmon Resonance Sensing with Nanoparticles and Thin Films: Nanomaterials, Surface Chemistry, and Hybrid Plasmonic Techniques. Anal. Chem. 2011, 83, 80578062.

2. Lamer, V.K.; Dinegar, R.H. Theory, Production and Mechanism of Formation of Monodispersed Hydrosols. J. Am. Chem. Soc. 1950, 72, 4847-4854.

3. Yagyu, H.; Sugano, K.; Hayashi, S.; Tabata, O. Micropowder Blasting using Nanoparticles Dispersed Polymer Mask for Rapid Prototyping of Glass Chip. J. Micromech. Microeng. 2005, 15, 1236-1241. 